FR-PHENO-2011-011,

DESY 11-116

\title{
A Simplified Scheme for GUT-inspired Theories with Multiple Abelian Factors
}

\author{
F. BRAAM ${ }^{1 a}$ AND J. ReUteR ${ }^{2 b}$ \\ ${ }^{a}$ Albert-Ludwigs-Universität Freiburg, Physikalisches Institut, Hermann-Herder-Str. 3, D-79104 \\ Freiburg, GERMANY \\ ${ }^{b}$ DESY Theory Group, Notkestr. 85, D-22603 Hamburg, GERMANY
}

\begin{abstract}
Grand Unified Theories often involve additional Abelian group factors apart from the standard model hypercharge, that generally lead to loop-induced mixing gauge kinetic terms. In this letter, we show that at the one-loop level this effect can be avoided in many cases by a suitable choice of basis in group space and present a general scheme for the construction of this basis. In supersymmetric theories however, a residual mixing in the soft SUSY breaking gaugino mass terms may appear. We generalize the renormalization group equations for the gaugino mass terms to account for this effect. In a further calculation we also present the necessary adjustments in the renormalization group equations of the trilinear soft breaking couplings and the soft breaking scalar mass squares.
\end{abstract}

\footnotetext{
${ }^{1}$ felix.braam@physik.uni-freiburg.de

2 juergen.reuter@desy.de
} 
The renormalization group equations (RGEs) describe the dependence of the coupling constants on the choice of the renormalization scale $\mu$, which is commonly translated into an energy dependence, as the perturbative series usually converges best if one chooses $\mu$ to be of the order of the characteristic energy scale of a given process. In (supersymmetric) grand unified model building [1/2[3/4/5]6] these equations constitute the framework which is employed to derive the potential unification of the gauge interactions into one fundamental force. They also describe the evolution of all other Lagrangian parameters (including the soft supersymmetry breaking parameters mediated to the "visible" sector through some mechanism at high scales) from a high unification scale down to the energy scales accessible to current collider experiments.

The RGEs for a (supersymmetric) model with an arbitrary semi-simple gauge group augmented by at most one $U(1)$ gauge group were given in [7/89|10] ([11/12 13]13]). We present a way of treating the case with several Abelian gauge groups, including a consistent generalization of the one-loop SUSY RGEs from [13] in the case, where a mixing of gauge kinetic terms at the tree-level does not occur, i.e.

$$
\kappa F_{\mu \nu}^{i} F^{\mu \nu, j}=0 \quad \forall i \neq j .
$$

In this situation we will show that the general concept presented in [1415] which has recently been applied to the complete set of SUSY two-loop RGEs in [16], where an additional coupling parametrizing the mixing is introduced, can be simplified considerably at the one-loop level by an appropriate choice of basis for the $U(1)$ groups.

First, we specify the type of models in which condition (11) holds, i.e. where our formalism is applicable: Consider some potentially multi-scale symmetry breaking scenario:

$$
\left.\mathcal{G}_{N}^{(0)} \stackrel{\Lambda}{\rightarrow} \mathcal{G}_{N-d}^{(1)}\right|_{d \geq 3} \times U(1)^{d-1} \rightarrow \mathrm{SM}
$$

where $\mathcal{G}_{N}^{(0)}$ denotes a simple 1 Lie group of rank $N$ and $\mathcal{G}_{N^{\prime}}^{(i)}$ an arbitrary semi-simple non-Abelian subgroup of it. This implies, that condition (1) holds at $\Lambda$ for all $U(1)$ groups in $\mathcal{G}_{N-d}^{(1)} \times U(1)^{2}$, as they all originate from non-Abelian gauge multiplets above $\Lambda$. A term as in Eq. (1) would have to necessarily arise from a matching condition at scale $\Lambda$

$$
\kappa G_{\mu \nu, 1} G_{2}^{\mu \nu} \rightarrow \kappa^{\prime} F_{\mu \nu, 1} F_{2}^{\mu \nu}
$$

with $G_{\mu \nu}$ being a non-Abelian field-strengh tensor, which istself is not gauge invariant. So the left-hand side of Eq. (3) is forbidden by the gauge symmetry.

Our argumentation holds if there are intermediate symmetry breaking steps above $\Lambda$, with arbitrary semi-simple gauge groups, as long as the rank $N$ is preserved and the matter content still fills complete multiplets of $\mathcal{G}_{N}^{(0)}$. At the tree-level, there cannot appear mixing terms in the course of symmetry

\footnotetext{
${ }^{1} \mathcal{G}_{N}^{(0)}$ does not necessarily have to be simple as long as the particle content can be assembled into complete multiplets of a simple Lie group and there are no mixing gauge kinetic terms at the tree-level.
} 
breaking, for the same reason as in Eq. (3). Furthermore the one-loop corrections to $\kappa^{\prime}$ in any intermediate phase vanish, as the trace over complete representations of the full GUT group vanishes for products of different generators:

$$
\operatorname{tr}\left[T^{A} T^{B}\right]=0 \quad \forall A \neq B .
$$

At the breaking scale $\Lambda$, a mixing among the $U(1)$ gauge-kinetic terms may be induced via quantum corrections from matter of representations made incomplete by the symmetry breaking. Typical examples for such scenarios can be found in [17/18 and arise e.g. in GUT breaking chains like $E_{6} \rightarrow S O(10) \times U(1)$ or $E_{6} \rightarrow S U(5) \times U(1)^{2}$.

We will now develop a scheme for constructing the basis for the $U(1)$ gauge groups, such that the mixing induced at the one-loop level can be avoided in the $\left.\mathcal{G}_{N-d}^{(3)}\right|_{d \geq 3} \times U(1)^{d-1}$ phase.

At $\Lambda$, we demand the gauge covariant derivative to be continuous:

$$
\left.D_{\mu}^{i}\right|_{\Lambda}=\left.D_{\mu}^{i+1}\right|_{\Lambda}
$$

From this we obtain a system of linear equations for the couplings and the charges of the new gauge group as functions of the corresponding parameters of the mother group:

$$
\begin{array}{rlcl}
g_{1}^{\prime} Q_{1}^{\prime} & =\lambda_{1,1} g_{1} Q_{1} & +\ldots & +\lambda_{1, d} g_{d} Q_{d} \\
\vdots & & \vdots \\
g_{d-1}^{\prime} Q_{d-1}^{\prime} & =\lambda_{d-1,1} g_{1} Q_{1} & +\ldots & +\lambda_{d-1, d} g_{d} Q_{d} \\
g_{d}^{\prime} Q_{d}^{\prime} & =\lambda_{d, 1} g_{1} Q_{1} & +\ldots & +\lambda_{d, d} g_{d} Q_{d} .
\end{array}
$$

Here, $U(1)_{i}^{\prime} i=1, \ldots, d-1$ are the remaining unbroken groups in $\left.\mathcal{G}_{N-d}^{(3)}\right|_{d \geq 3} \times U(1)^{d-1}$, and $U(1)_{d}^{\prime}$ corresponds to the broken Cartan generator of the non-Abelian gauge group, respectively. In Eq. (5) there are $d^{2}+2 d$ free parameters $\left\{g_{i}^{\prime}, Q_{i}^{\prime}, \lambda_{i, j}\right\}$ which can be uniquely determined (up to signs) by applying the following constraints:

1. The vacuum expectation value breaking the symmetry at scale $\Lambda$ is not charged under the unbroken $U(1)$ groups:

$$
Q_{i}^{\prime}\left\langle H_{\Lambda}\right\rangle=0 \quad \text { for } i=1, \ldots, d-1
$$

2. The broken and unbroken generators are normalized according to the Dynkin index of some complete GUT representation $R, S(R)$ (no summation over $i$ ):

$$
\sum_{\alpha \in R} Q_{i}^{\prime}(\alpha) Q_{i}^{\prime}(\alpha)=S(R) \quad \text { for } i=1, \ldots, d
$$

3. Vanishing mixing at the one-loop level:

$$
\begin{gathered}
0=\frac{1}{3} \sum_{R^{\phi}} \sum_{\alpha \in R^{\phi}} Q_{i}^{\prime}(\alpha) Q_{j}^{\prime}(\alpha)+\frac{2}{3} \sum_{R^{\psi}} \sum_{\beta \in R^{\psi}} Q_{i}^{\prime}(\beta) Q_{j}^{\prime}(\beta) \\
\text { for } i \neq j ; i=1, \ldots, d-1 ; j=1, \ldots, d-1,
\end{gathered}
$$

where $R^{\phi}$ and $R^{\psi}$ denote representations of scalars and Weyl fermions, respectively. 
4. Corresponding transformations of the gauge fields are orthogonal:

$$
\left(A_{1}^{\prime \mu}, \ldots, A_{d}^{\prime \mu}\right)=\left(A_{1}^{\mu}, \ldots, A_{d}^{\mu}\right) \lambda^{T}, \quad \text { with } \quad \lambda_{i j} \lambda_{k j}=\delta_{i k}
$$

A suitable framework to construct the charge operators in (5) and (6) is the concept of projection matrices in the weight space of the gauge groups, as presented in [19. The representations to sum over in the constraints 2 . and 3 . as well as the field with non-trivial vacuum expectation value breaking the symmetry in constraint 1 . can then be represented by their corresponding weights.

Applying this procedure at the scale where the rank of the gauge group is reduced for the first time in the chain of symmetry breakings ensures that there does not occur any mixing among gauge-kinetic terms at the one-loop level. If there are subsequent symmetry breaking mechanisms leading again to several Abelian gauge groups, the procedure can be applied repeatedly. The advantage of this scheme is, that one can still apply the one-loop RGEs as given in [7/8/9|10] [13] for gauge and superpotential couplings without any changes.

Although, by this procedure one can avoid a mixing in the gauge kinetic term, a remnant shows up for softly broken supersymmetric models, unless the corresponding gaugino masses at the breaking scale are degenerate, as

$$
\mathcal{L}_{M_{g}}=\overline{\tilde{\chi}}_{i} M_{i i} \tilde{\chi}_{i}=\overline{\tilde{\chi}}^{a} \underbrace{\lambda_{i}^{a} M_{i j} \lambda_{j}^{b}}_{\equiv M^{a b}} \tilde{\chi}^{b} \quad \Rightarrow \quad M^{a b} \neq \operatorname{diag}, \text { unless } M_{i i} \equiv M \forall i .
$$

At first glance, non-degenerate gaugino masses seem rather artificial (though the SUSY-breaking mechanism might not be completely $U(1)$-blind). Note however, that they naturally appear in the multi-scale models mentioned above, as $U(1)$ gaugino masses can evolve differently between intermediate scale above $\Lambda$, as well as in SUSY-breaking mechanisms sensitive to the beta function of the gauge group under consideration like AMSB [20]. Another example where non-degenerate gaugino mass terms could arise are mixed mediation mechanisms like e.g. mirage mediation [21].

In the following, we will present the generalized RGEs in the $\overline{\mathrm{DR}}$ scheme $[22$ at the one-loop level for soft SUSY breaking terms, accounting for this effect. We use the conventions and nomenclature of [13. In the absence of $U(1)$ mixing the one-loop RGEs for the soft-breaking gaugino masses are given by $[13$.

$$
\frac{d}{d t} M_{a}=\frac{2}{16 \pi^{2}} \beta_{a} g_{a}^{2} M_{a}
$$

with

$$
\beta_{a}=S(R)-3 C(G)
$$

where $S(R)$ is the Dynkin index summed over all chiral superfields, and $C(G)$ the quadratic casimir of the adjoint representation, respectively. The logarithm of the ratio of scales is denoted as $t=\log \mu / \mu_{0}$. In the presence of gaugino mass mixing terms, we have to extend Eq. (7) in order to describe the running of the full gaugino mass matrix:

$$
\frac{d}{d t} M_{a b}=\frac{1}{16 \pi^{2}}\left(\beta_{a} g_{a}^{2} M_{a b}+\beta_{b} g_{b}^{2} M_{a b}\right) .
$$


These contributions arise from the following diagrams
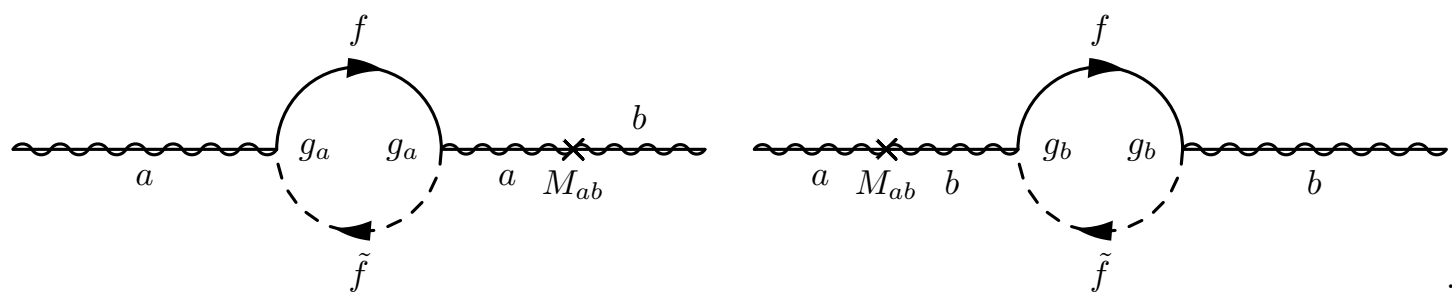

Note, that the running of the diagonal entries in the gaugino mass matrix are not altered in the presence of mixed mass terms, as diagrams like

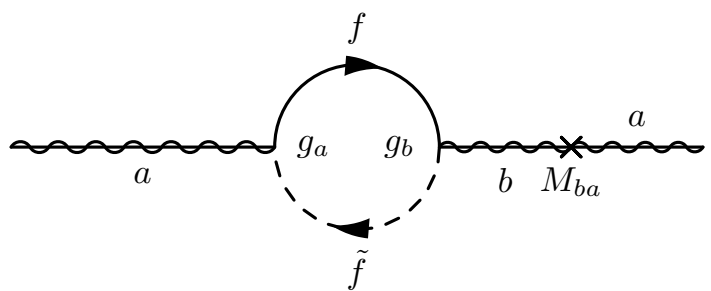

are by construction canceled out after rotating to the new $U(1)$ basis.

Besides this effect on the running of the full gaugino mass matrix, there is also a modification of the RGEs for the trilinear soft breaking parameters by non-diagonal gaugino mass terms. The new diagrams contributing to the running of the trilinear terms are of the form:

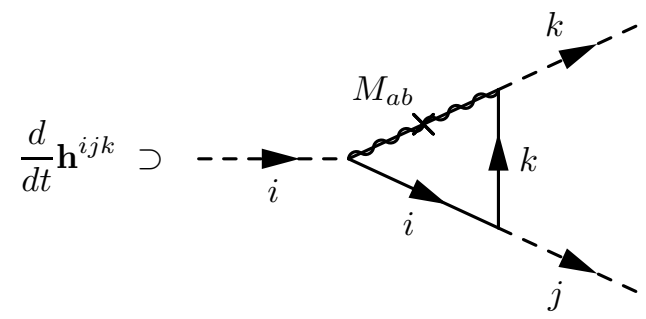

The corresponding renormalization group equations for the soft trilinear terms now read

$$
\begin{gathered}
\frac{d \mathbf{h}^{i j k}}{d t}=\frac{1}{16 \pi^{2}}\left[\frac{1}{2} \mathbf{h}^{i j l} \mathbf{Y}_{l p q} \mathbf{Y}^{p q k}+\mathbf{Y}^{i j l} \mathbf{Y}_{l p q} \mathbf{h}^{p q k}+2\left(\delta_{a b} \mathbf{h}^{i j k}-2 M_{a b} \mathbf{Y}^{i j k}\right) g_{a} g_{b} C_{a b}(k)\right] \\
+(k \leftrightarrow i)+(k \leftrightarrow j),
\end{gathered}
$$

with

$$
C_{a b}(k)=\left[\begin{array}{lll}
C_{a}(k) & \text { if } & a=b \\
Q_{k}^{a} Q_{k}^{b} & \text { if } & a \neq b
\end{array} .\right.
$$

The first line has to be applied for non-Abelian groups, while the second line accounts for $U(1)$ factors.

Finally, for the scalar mass squared terms, the generalization of the RGE result calculated in [13], adjusted to take $U(1)$ mixing into account, reads:

$$
\begin{gathered}
\frac{d}{d t}\left(\mathbf{m}^{2}\right)_{j}^{i}=\frac{1}{16 \pi^{2}}\left[\frac{1}{2} \mathbf{Y}_{j p q} \mathbf{Y}^{p q n}\left(\mathbf{m}^{2}\right)_{n}^{i}+\frac{1}{2} \mathbf{Y}^{i p q} \mathbf{Y}_{p q n}\left(\mathbf{m}^{2}\right)_{j}^{n}+2 \mathbf{Y}_{j p q} \mathbf{Y}^{i p n}\left(\mathbf{m}^{2}\right)_{n}^{q}+\mathbf{h}_{j p q} \mathbf{h}^{i p q}\right. \\
\left.-8 \delta_{j}^{i}\left|M_{a b}\right|^{2} g_{a} g_{b} \mathcal{C}_{a b}(j)+2 g_{\beta}^{2} \delta_{j}^{i} Q_{j}^{\beta}\left(\delta_{k}^{l} Q_{k}^{\beta}\left(\mathbf{m}^{2}\right)_{l}^{k}\right)\right]
\end{gathered}
$$


where in the last term the index $\beta$ runs over all $U(1)$ factors.

\section{Conclusions:}

In this letter, we studied a class of (supersymmetric) GUT models, where $U(1)$ mixing forbidden at tree-level can occur at the (one-)loop level and defined a generic matching scheme for the couplings at intermediate thresholds by constructing a suitable choice of basis. Such a basis avoids - at the oneloop level - the mixing of the gauge couplings and completely diagonalizes the $U(1)$ vector (super-) fields (in the absence of gaugino mass non-degeneracy). In that case, using this specific basis allows to use the setup in [813] without any changes. In phenomenologically interesting SUSY GUT models, however, gaugino masses at some high or intermediate scale could be non-degenerate, mostly by means of running or through some explicit construction. For such a case, gaugino masses can not longer be simultaneously diagonalized. Consequently, we gave the modifications for the renormalization group equations for the gaugino masses, the trilinear soft breaking terms and the sfermion soft mass squared terms at the one-loop order.

\section{Acknowledgements}

We thank L. Basso, S. Dittmaier, C. Horst, F. Jörder, A. Knochel, F. Staub, and J.J. van der Bij for stimulating remarks and discussions. This work has been supported by the German Research Council (DFG) under Grant No. RE/2850/1-1 as well as by the Ministery for Research and Culture (MWK) of the German state Baden-Württemberg, and has also been partially supported by the DFG Graduiertenkolleg GRK 1102 "Physics at Hadron Colliders".

Note added: As this paper has been finished there has been a similar work calculating the 1- and 2-loop RGEs for two mixing $U(1)$ s in a non-diagonal basis, [16].

\section{References}

[1] H. Georgi, S. L. Glashow, Phys. Rev. Lett. 32, 438-441 (1974).

[2] J. C. Pati, A. Salam, Phys. Rev. D10, 275-289 (1974).

[3] H. Fritzsch, P. Minkowski, Annals Phys. 93, 193-266 (1975).

[4] Y. Achiman, B. Stech, Phys. Lett. B77, 389 (1978).

[5] J. Wess, J. Bagger, "Supersymmetry and supergravity," Princeton, USA: Univ. Pr. (1992) 259 p.

[6] J. Terning, "Modern supersymmetry: Dynamics and duality," Oxford, UK: Clarendon (2006) 324 p.

[7] D. R. T. Jones, Nucl. Phys. B75, 531 (1974). 
[8] M. E. Machacek, M. T. Vaughn, Theory. 3. Scalar Quartic Couplings," Nucl. Phys. B249, 70 (1985).

[9] M. E. Machacek, M. T. Vaughn, Theory. 2. Yukawa Couplings," Nucl. Phys. B236, 221 (1984).

[10] M. E. Machacek, M. T. Vaughn, Theory. 1. Wave Function Renormalization," Nucl. Phys. B222, 83 (1983).

[11] D. R. T. Jones, Approximation," Nucl. Phys. B87, 127 (1975).

[12] D. R. T. Jones, L. Mezincescu, Phys. Lett. B136, 242 (1984).

[13] S. P. Martin and M. T. Vaughn, Phys. Rev. D 50, 2282 (1994) [Erratum-ibid. D 78, 039903 (2008)] arXiv:hep-ph/9311340.

[14] F. del Aguila, G. D. Coughlan and M. Quiros, Nucl. Phys. B 307 (1988) 633 [Erratum-ibid. B 312 (1989) 751].

[15] A. Ferroglia, A. Lorca and J. J. van der Bij, Annalen Phys. 16 (2007) 563 [arXiv:hep-ph/0611174].

[16] R. Fonseca, M. Malinsky, W. Porod, F. Staub, arXiv:1107.2670 [hep-ph]].

[17] F. Braam, A. Knochel and J. Reuter, JHEP 1006, 013 (2010), arXiv:1001.4074 [hep-ph]].

[18] W. Kilian, J. Reuter, Phys. Lett. B642, 81-84 (2006). hep-ph/0606277.

[19] R. Slansky, Phys. Rept. 79 (1981) 1.

[20] L. Randall, R. Sundrum, Nucl. Phys. B557, 79-118 (1999). hep-th/9810155]; G. F. Giudice, M. A. Luty, H. Murayama, R. Rattazzi, JHEP 9812, 027 (1998). hep-ph/9810442.

[21] S. Kachru, R. Kallosh, A. D. Linde, S. P. Trivedi, Phys. Rev. D68, 046005 (2003). hep-th/0301240]; K. Choi, A. Falkowski, H. P. Nilles, M. Olechowski, S. Pokorski, JHEP 0411, 076 (2004). hep-th/0411066].

[22] W. Siegel, Phys. Lett. B84, 193 (1979); D. M. Capper, D. R. T. Jones, P. van Nieuwenhuizen, Nucl. Phys. B167, 479 (1980); J. A. Aguilar-Saavedra et al., Eur. Phys. J. C46, 43-60 (2006). hep-ph/0511344. 